\title{
Mice Lacking Functional Fas Death Receptors Are Protected from Kainic Acid-Induced Apoptosis in the Hippocampus
}

\author{
Miren Ettcheto - Felix Junyent • Luisa de Lemos • Merce Pallas • Jaume Folch • \\ Carlos Beas-Zarate • Ester Verdaguer • Raquel Gómez-Sintes • José J. Lucas • \\ Carme Auladell $\cdot$ Antoni Camins
}

Received: 30 May 2014 / Accepted: 25 July 2014 / Published online: 15 August 2014

(C) Springer Science+Business Media New York 2014

\begin{abstract}
The Fas receptor (FasR)/Fas ligand (FasL) system plays a significant role in the process of neuronal loss in neurological disorders. Thus, in the present study, we used a real-time PCR array focused apoptosis (Mouse Apoptosis RT ${ }^{2}$ PCR Array) to study the role of the Fas pathway in the apoptotic process that occurs in a kainic acid (KA) mice experimental model. In fact, significant changes in the transcriptional activity of a total of 23 genes were found in the hippocampus of wild-type C57BL/6 mice after $12 \mathrm{~h}$ of KA treatment compared to untreated mice. Among the upregulated genes, we found key factors involved in the extrinsic apoptotic pathway, such as thf, fas and fas $L$, and also in caspase genes (caspase-4, caspase-8 and caspase-3). To discern the importance of the FasR/FasL pathway, mice lacking the functional Fas death receptor $(l p r)$ were also treated with KA. After $24 \mathrm{~h}$ of neurotoxin treatment, $l p r$ mice exhibited a reduced number of apoptotic positive cells, determined by the
\end{abstract}

Carme Auladell and Antoni Camins are senior co-authors.

Miren Ettcheto and Felix Junyent contributed equally to the manuscript

M. Ettcheto $\cdot$ F. Junyent $\cdot$ L. de Lemos $\cdot$ M. Pallas $\cdot$ A. Camins $(\triangle)$ Unitat de Farmacologia i Farmacognòsia Facultat de Farmàcia, Institut de Biomedicina (IBUB), Universitat de Barcelona, Avda/ Diagonal 643, E-08028 Barcelona, Spain

e-mail: camins@ub.edu

M. Ettcheto $\cdot$ F. Junyent $\cdot$ L. de Lemos $\cdot$ M. Pallas $\cdot$ J. Folch $\cdot$

R. Gómez-Sintes · J. J. Lucas · C. Auladell · A. Camins

Networking Research Center on Neurodegenerative Diseases

(CIBERNED), Instituto de Salud Carlos III, Madrid, Spain

J. Folch

Unitat de Bioquímica, Facultat de Medicina i Ciències de la Salut,

Universitat Rovira i Virgili, Reus, Tarragona, Spain

\section{Beas-Zarate}

Laboratorio de Neurobiología Celular y Molecular, División de

Neurociencias, CIBO, IMSS, Guadalajara, Mexico terminal deoxynucleotidyl transferase dUTP nick end labelling (TUNEL) method in different regions of the hippocampus, when compared to wild-type mice. In addition, treatment of $l p r$ mice with KA did not produce significant changes in the transcriptional activity of genes related to apoptosis in the hippocampus, either in the fas and fas ligand genes or in caspase- 4 and caspase- 8 and the executioner caspase- 3 genes, as occurred in wild-type C57BL/6 mice. Thus, these data provide direct evidence that Fas signalling plays a key role in the induction of apoptosis in the hippocampus following KA treatment, making the inhibition of the death receptor pathway a potentially suitable target for excitotoxicity neuroprotection in neurological conditions such as epilepsy.

Keywords Kainic acid $\cdot$ Apoptosis $\cdot$ FAS $\cdot$ Neuroprotection . Microglia $\cdot$ Caspase-3

C. Beas-Zarate

Laboratorio de Desarrollo y Regeneración Neural, Instituto de Neurobiología, Departamento de Biología Celular y Molecular, CUCBA, Universidad de Guadalajara, Guadalajara, Jalisco, Mexico

R. Gómez-Sintes · J. J. Lucas

Center for Molecular Biology "Severo Ochoa" (CBMSO)

CSIC/UAM, Madrid, Spain

E. Verdaguer $\cdot$ C. Auladell

Departament de Biologia Cel•lular, Facultat de Biologia, Universitat de Barcelona, Barcelona, Spain 


\section{Introduction}

It is widely known that the excitotoxic process is a contributing factor to acute neuronal damage in most chronic neurodegenerative diseases, such as Alzheimer's disease, Parkinson's disease and Huntington's disease [1-3]. Previous reports have demonstrated that the administration of kainic acid (KA) in rodents, an analogue of the excitotoxin glutamate, produces epileptic seizures [4-9]. The greatest abundance of kainate receptors is present in the hippocampal CA3 region, and their activation results in neuronal loss of selective populations in the hippocampus and other brain structures. Moreover, numerous studies have demonstrated that neuronal loss observed in this excitotoxic model involves, at least in part, apoptotic cell death $[10,11]$. Likewise, KA can stimulate CA3 neurons directly through stimulation of their own KA receptors and also indirectly by favouring glutamate release secondary to KA stimulation of mossy fibres [4].

One important point is the characterisation of the mechanism involved in KA-induced neurodegeneration. Thus, a large number of studies have been conducted to identify the potential biochemical pathways involved in KA-induced apoptosis, such as oxidative stress, cell cycle re-entry and calpain/cdk5 activation [8, 10-16]. Programmed cell death is primarily mediated by the extrinsic or death receptor pathway, and the intrinsic or mitochondrial pathway, which converge to activate mitochondria $[17,18]$. This constitutes a key point because it favours the release of some proteins such as cytochrome c, Smac/Diablo and Omi/HrtA2 into the cytosol, which facilitates the activation of common executioner caspases (caspase-3/7) with the final cleavage of downstream targets that include DNA repair enzymes [19].

It has been previously shown that the extrinsic apoptotic pathway induces apoptosis in lymphoid cells and in neuronal cells deprived of critical survival factors and also in models of excitotoxicity [20]. The Fas receptor is known to induce apoptosis by binding to FasL. Thus, the Fas ligand (FasL) and TNF-related apoptosis-inducing ligand (TRAIL) are upregulated following experimental KA administration to rodents [21-23]. In addition, previous studies have shown that the binding of the Fas ligand to FasR triggers the formation of a death-inducing signalling complex (DISC), which consists of FasL, FasR, the adapter protein FADD (Fas-associated death domain protein), and the TNFR1-associated death domain protein (TRADD) to induce the activation of caspase-8, a protease which then activates downstream effector caspase3 , which in turn is responsible for apoptosis. Thus, both extrinsic and intrinsic apoptotic pathways converge to activate common executioner caspases (caspase-3/7) with the final cleavage of downstream targets. Inhibition of Fas-mediated apoptosis is regulated by the FLICE-inhibitory protein (FLIP), the structural homologue of procaspase-8 [24-29]. lpr mice have alterations in the Fas receptor gene due to the insertion of a retrotransposon that causes disruption in its transcription [30]. Attenuation of the Fas signalling system has been related to neurological damage protection in various experimental models of pathophysiological diseases such as ischemia and multiple sclerosis [27, 29-33]. More precisely, $l p r$ mice were protected from the neurotoxicity mediated by the neurotoxin MPTP (1-methyl-4-phenyl-1,2,3,6tetrahydropyridine), which causes permanent symptoms of Parkinson's disease [34, 35]. Interestingly, in post-mortem brains of Parkinson's patients, the levels of Fas are increased in the nigrostriatal region [36]. In addition, deletions of the Fas receptor reduced lesion size and improved brain function after a traumatic brain injury, confirming the role of these signalling pathways in the pathogenesis of delayed brain damage [7, 25]. All these data raised the possibility that Fas signalling activated in different pathologies may render neurons vulnerable to dying $[31,37]$.

Furthermore, recent studies have specifically exemplified the role of Fas in Alzheimer's disease (AD) neurodegeneration $[28,38-40]$. It has been suggested that $\beta$-amyloid can induce neuronal death via processes of apoptosis [40, 41]. Similarly, it has been demonstrated that neurons in the AD brain and neuronal cultures treated with beta-amyloid show a significant increase in Fas ligand expression and immunoreactivity [42]. Moreover, cortical cultures of lpr mice that carry mutations leading to a loss of function of Fas genes are protected from beta-amyloid toxicity. These results, therefore, lend support to the involvement of this pathway in $\mathrm{AD}$ apoptosis.

Given the lack of direct evidence linking FasR with the intrinsic apoptotic pathway, we sought to clarify the role of apoptosis mediated by Fas signalling after KA treatment. The present study examined the impact of KA administration in C57BL/6 Fas-deficient (lpr) mice, which exhibit low levels of Fas receptor expression [43, 44]. To investigate whether FasL signalling is also relevant to the neurotoxic process mediated by KA, we analysed the hippocampal apoptotic cell death after intraperitoneal KA administration in Fas receptordeficient mice ( $l p r$ mice). Moreover, a study of apoptotic gene expression was performed in the hippocampus and here we demonstrated that loss of Fas receptor activity ( $l p r$ mice) is neuroprotective against excitotoxicity.

\section{Material and Methods}

Animals

Wild-type C57BL/6 mice were obtained from Harlan Laboratories or the Jackson Laboratory. Fas-deficient $l p r$ mice (C57BL/6 background) were obtained from Jackson Laboratory (B6.MRL-FasLpr/J, stock number 000482). All mice 
were housed at the Centro Biología Molecular Severo Ochoa in Madrid. Mice were housed four per cage with food and water available ad libitum and maintained in a temperaturecontrolled environment on a 12-h light/12-h dark cycle with light onset at $7 \mathrm{am}$. All experiments were performed under a protocol approved by the CSIC ethical committee.

\section{Kainic Acid Treatment and Sample Preparation}

A total of 40 mice were used for the present study. Six-weekold mice were treated with a single intraperitoneal (i.p.) dose (30 mg/kg) of KA (Sigma-Aldrich, St. Louis, MO, USA) or with saline solution for controls. For RNA analysis, 10 wildtype C57BL/6 mice and $10 \mathrm{lpr}$ mice were used. Five mice from each group were used as controls and were treated with KA and killed after $12 \mathrm{~h}$; the hippocampus was then excised and frozen. For immunochemistry and immunofluorescence experiments, we used 10 wild-type C57BL/6 mice and $10 \mathrm{lpr}$ mice. Five from each group served as control and were injected with KA and killed after $24 \mathrm{~h}$.

All mice were anaesthetised by injection (i.p.) of pentobarbital $80 \mathrm{mg} / \mathrm{kg}$. After that, the mice were perfused with $40 \mathrm{~g} / \mathrm{L}$ paraformaldehyde in $0.1 \mathrm{~mol} / \mathrm{L}$ phosphate buffer $(\mathrm{PB})$ and the brains were removed and post-fixed for $24 \mathrm{~h}$. The brains were subsequently rinsed in the same solution with $300 \mathrm{~g} / \mathrm{L}$ sucrose for $24 \mathrm{~h}$ and then frozen. Coronal sections of $30 \mu \mathrm{m}$ were obtained by a Cryostat (Leica Microsystems, Wetzlar, Germany).

\section{$\mathrm{RT}^{2}$ Profiler $^{\mathrm{TM}}$ PCR Array}

The Mouse Apoptosis RT ${ }^{2}$ Profiler PCR Array (PAMM012A, Biosciences, Frederick, MD, USA) profiles the expression of 84 genes involved in apoptosis together with five housekeeping genes. Controls for genomic DNA contamination and for the efficiency of the RT-PCR and PCR were also included in the array and were evaluated after each run. One microgram of total RNA was reverse transcribed in a final reaction mix of $20 \mu \mathrm{L}$ using the $\mathrm{RT}^{2}$ First Strand Kit according to the manufacturer's instructions. For one 96-well plate of the PCR array, $1,100 \mu \mathrm{L}$ of PCR master mix containing

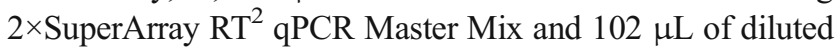
cDNA were prepared, and aliquots of $10 \mu \mathrm{L}$ were added to each well. The PCR was performed on the LightCycler 480 (Roche Applied Science, Manheim, Germany), and universal cycling conditions $\left(10 \mathrm{~min}\right.$ at $95^{\circ} \mathrm{C}, 15 \mathrm{~s}$ at $95^{\circ} \mathrm{C}, 1 \mathrm{~min}$ at $60{ }^{\circ} \mathrm{C}$ for 40 cycles) were used. Expression of each gene was normalised to the mean $\mathrm{Ct}$ for five housekeeping genes in the PCR array ( $\beta$-glucuronidase, hypoxanthine phosphoribosyltransferase, heat shock protein $90 \alpha$, glyceraldehyde-3-phosphate dehydrogenase and $\beta$-actin). Relative fold change in expression was calculated using the $\Delta \Delta \mathrm{Ct}$ method, and the values were expressed as $2-\Delta \Delta \mathrm{Ct}$.
The statistical calculation was based on the web-based program of RT ${ }^{2}$ Profiler ${ }^{\mathrm{TM}}$ PCR Array Data Analysis. Significant changes in gene expression between KA-treated mice and untreated controls were assumed as a fold change $\geq 1.5$ and $\leq 0.6$, with a $P$ value $<0.05$. The experiments were repeated three times.

RNA Extraction and Real-Time Reverse Transcription-Polymerase Chain Reaction (qRT-PCR)

Total RNA was isolated from mouse hippocampus using TRIzol (Invitrogen, Carlsbad, CA, USA) followed by chloroform, according to the manufacturer's protocol. RNA concentration was measured using a NanoDrop ${ }^{\mathrm{TM}} 1000$ Spectrophotometer (Thermo Scientific, MA, USA).

As a general procedure, $1 \mu \mathrm{g}$ of total RNA was reverse transcribed using a high-capacity cDNA reverse transcription kit (Applied Biosystems, Carlsbad, CA, USA). The same amounts of cDNA were subsequently used for quantitative real-time PCR using SYBR Green ${ }^{\circledR}$ PCR Master Mix and performed on the StepOnePlus ${ }^{\text {TM }}$ Real-Time PCR System (Applied Biosystems, Carlsbad, CA, USA). All samples were run in triplicate, and expression values were normalised to the housekeeping $\beta$-actin in the same reaction. Relative normalised mRNA levels were calculated using the $\Delta \Delta \mathrm{Ct}$ method. Primers used for quantitative real-time PCR are shown in Table 1. Genespecific primers corresponding to the PCR targets on the PCR $\mathrm{RT}^{2}$ array were designed using Primer Express ${ }^{\circledR}$ Software v2.0 (Applied Biosystems, Carlsbad, CA, USA).

\section{Fluoro-Jade Staining}

Neurodegeneration was assessed using Fluoro-Jade B (Chemicon Europe Ltd). Slides were rinsed with phosphatebuffered saline (PBS), followed by two washes in distilled water. Afterwards, slides were immersed in $0.6 \mathrm{~g} / \mathrm{L}$ potassium permanganate $\left(\mathrm{KMnO}_{4}\right)$ for $15 \mathrm{~min}$ in the dark. Then, after two washes in distilled water, the slides were transferred to the staining solution containing $0.1 \mathrm{~mL} / \mathrm{L}$ acetic acid and $0.004 \mathrm{~mL} / \mathrm{L}$ of the fluorochrome Fluoro-Jade B for $30 \mathrm{~min}$ in the dark. Slides were rinsed in distilled water, dried, and then submerged directly into xylene and mounted in DPX medium. Slides were analysed using epifluorescence microscopy (Olympus BX61, Olympus, Barcelona, Spain). To determine the positive cell number for Fluoro-Jade B, we counted the cells in each area from serial sections of hippocampus from each mouse brain.

\section{TUNEL Assay}

DNA damage was assessed using terminal deoxynucleotidyl transferase dUTP nick end labelling (TUNEL) staining method. TUNEL staining was performed as described in the In situ 
Table 1 Significant changes in transcriptional activity of genes included in the Mouse Apoptosis RT ${ }^{2}$ Profiler PCR Array, PAMM-012A, and from the hippocampus of KA-treated C57/B16 wild-type mice, at $12 \mathrm{~h}$. The administration of this neurotoxin caused significant changes in the expression of 23 genes: 17 genes resulted significantly up-regulated; the most significant up-regulation corresponded to the $\operatorname{tnf}$ gene that showed a fold change of 15.95, and the lowest significant gene activation levels corresponded to hells, with a fold change of 1.5. Another six genes resulted down-regulated. Significant changes in gene expression between KA-treated mice and untreated controls were assumed as a fold change $\geq 1.5$ and $\leq 0.6$, with a $P$ value $<0.05$. The experiments were repeated three times

\begin{tabular}{|c|c|c|c|c|}
\hline GenBank number & Gene symbol & Gene name & Fold change & $P$ value \\
\hline NM_013693 & $\operatorname{Tnf} \alpha$ & Tumour necrosis factor & 15.95 & 0.0017 \\
\hline NM_013863 & Bag3 & Bcl2-associated athanogene 3 & 9.03 & 0.0002 \\
\hline NM_011609 & Tnfr1 & Tumour necrosis factor receptor superfamily, member 1a & 6.51 & 0.0004 \\
\hline NM_007987 & Fas & Fas (TNF receptor superfamily member 6) & 3.53 & 0.0001 \\
\hline NM_008562 & Mcl1 & Myeloid cell leukaemia sequence 1 & 3.47 & 0.0001 \\
\hline NM_009805 & Cflar & CASP8 and FADD-like apoptosis regulator & 2.91 & 0.0003 \\
\hline NM_007609 & Casp4 & Caspase- 4 , apoptosis-related cysteine peptidase & 2.21 & 0.0002 \\
\hline NM_010177 & Fasl & Fas ligand (TNF superfamily, member 6) & 2.00 & 0.0017 \\
\hline NM_010736 & $\mathrm{LtBr}$ & Lymphotoxin B receptor & 1.99 & 0.0001 \\
\hline NM_009740 & $\mathrm{Bcl10}$ & B cell leukaemia/lymphoma 10 & 1.89 & 0.0336 \\
\hline NM_009422 & Traf2 & Tnf receptor-associated factor 2 & 1.83 & 0.0001 \\
\hline NM_138606 & Pim2 & Proviral integration site 2 & 1.78 & 0.0002 \\
\hline NM_009810 & Casp3 & Caspase-3 & 1.78 & 0.0086 \\
\hline NM_030693 & Atf5 & Activating transcription factor 5 & 1.73 & 0.0001 \\
\hline NM_172858 & Pak7 & P21 protein $(\mathrm{Cdc} 42 / \mathrm{Rac})$-activated kinase 7 & 1.62 & 0.0003 \\
\hline NM_009812 & Casp8 & Caspase- 8 & 1.54 & 0.0002 \\
\hline NM_008234 & Hells & Helicase, lymphoid specific & 1.50 & 0.0214 \\
\hline NM_009950 & Cradd & CASP2 and RIPK1 domain containing adaptor with death domain & -1.56 & 0.0001 \\
\hline NM_007702 & Cidea & Cell death-inducing DNA fragmentation, $\alpha$ subunit-like effector A & -1.56 & 0.0089 \\
\hline NM_001163138 & Card6 & Caspase recruitment domain family, member 6 & -1.61 & 0.0003 \\
\hline NM_173378 & Trp53bp2 & Transformation related protein 53 binding protein 2 & -1.69 & 0.0008 \\
\hline NM_030152 & Nol3 & Nuclear protein 3 (apoptosis repressor with CARD domain) & -1.88 & 0.0007 \\
\hline NM_080637 & Nme5 & Non-metastatic cells 5 & -2.34 & 0.0001 \\
\hline
\end{tabular}

Cell Death Detection Kit, TMR red (Roche Molecular Biochemicals, IN, USA) with minor modifications. Sections were rinsed with PBS and immersed in a permeabilisation solution $\left(0.1 \%\right.$ Triton X-100, $0.1 \%$ sodium citrate) for $2 \mathrm{~min}$ at $4{ }^{\circ} \mathrm{C}$. Then, slides were washed twice with PBS and incubated with TUNEL reaction mixture in a humidified atmosphere for $60 \mathrm{~min}$ at $37^{\circ} \mathrm{C}$ in the dark. Afterwards, slides were rinsed three times with PBS and then incubated with $0.1 \mu \mathrm{g} / \mathrm{mL}$ Hoechst 33258 (Sigma-Aldrich, St Louis, MO, USA) nuclear stain for $5 \mathrm{~min}$ in the dark. Following a final rinse with PBS, slides were mounted in Fluoromount medium (SigmaAldrich, St Louis, MO, USA) and analysed under a confocal fluorescence microscope (Leica TCS SP2 confocal microscope).

Immunohistochemistry

Free-floating coronal sections, $30 \mu \mathrm{m}$ thick, were rinsed in $0.1 \mathrm{~mol} / \mathrm{L} \mathrm{PB} \mathrm{pH} 7.2$ and treated with $5 \mathrm{~mL} / \mathrm{L} \mathrm{H}_{2} \mathrm{O}_{2}$ and $100 \mathrm{~mL} / \mathrm{L}$ methanol in PBS. After that, they were pre- incubated in a blocking solution $(100 \mathrm{~mL} / \mathrm{L}$ of foetal bovine serum (FBS), $2.5 \mathrm{~g} / \mathrm{L}$ of bovine serum albumin and $0.2 \mathrm{~mol} / \mathrm{L}$ of glycine in PBST, PBS with $5 \mathrm{~mL} / \mathrm{L}$ of Triton $\mathrm{X}-100)$. Then, sections were incubated overnight $(\mathrm{O} / \mathrm{N})$ at $4{ }^{\circ} \mathrm{C}$ with different primary antibodies: rabbit anti-GFAP (1:2,000; Dako, Glostrup, Denmark) and rabbit anti-Iba1 (1:1,000; Wako, Osaka, Japan), and sequentially incubated for $2 \mathrm{~h}$ with Alexa Fluor 594 goat anti-rabbit antibody (1:500; Invitrogen, Eugene, OR, USA). Sections were counterstained with $0.1 \mu \mathrm{g}$ / mL Hoechst 33258 (Sigma-Aldrich, St Louis, MO, USA) nuclear stain for $5 \mathrm{~min}$ in the dark. Immediately, sections were rinsed with $\mathrm{PBS}$ and mounted onto gelatinised slides with Fluoromount medium (Sigma-Aldrich, St Louis, MO, USA). Stained sections were examined under a confocal fluorescence microscope (Leica TCS SP2 confocal microscope).

\section{Statistical Analysis}

All experiments were conducted in triplicate, and the results were expressed as mean values \pm SEM. Differences are 
considered significant at $P<0.05$. $P$ values were determined by Student's $t$ test.

\section{Results}

Identification of the Expression of Apoptotic Genes in the Hippocampus of Wild-Type Mice, After Treatment with Kainic Acid

The administration of KA at a dose of $30 \mathrm{mg} / \mathrm{kg}$ in mice favours the onset of seizures and a variety of behavioural changes that have been previously described by a number of laboratories [7-9, 45]. The lpr mice compared to the wild type did not show an improvement in the number of seizures.

To assess whether apoptosis-related genes showed changes in expression in KA-treated mice at $12 \mathrm{~h}, 84$ genes were analysed using the real-time PCR array (Mouse Apoptosis $\mathrm{RT}^{2}$ Profiler PCR Array). The results indicated that administration of this neurotoxin caused significant changes in the expression of 23 genes (Table 1). Among them, a total of 17 genes were significantly up-regulated, whereas the transcriptional activity of six genes was below normal levels. The most significant up-regulation corresponded to the thf, which showed a fold change of 15,95 with $P$ value $=0.0017$ and the lowest significant gene activation levels corresponded to Hells, with a fold change of 1.5 with $P$ value $=0.0214$. In relation to this observation, the exposure of the wild-type strain to KA toxicity significantly activated genes related to the Fas signalling pathway: fas, fasl, and caspase-4, caspase-3 and caspase-8.

\section{Evaluation of Kainic Acid-Induced Neurotoxicity in the Hippocampus of Fas-Deficient lpr Mice}

The real-time PCR array results suggested an involvement of the Fas pathway in the apoptotic process occurring in the hippocampus of C57/B16 mice, so the next step was to evaluate the implication of this pathway in KA-induced neuronal death. Then, we decided to analyse the neurodegeneration induced in Fas-deficient $l p r$ mice following KA injection. After $24 \mathrm{~h}$ of treatment with KA, the neuronal death in hippocampal areas was assessed using the Fluoro-Jade B technique. Interestingly, brain sections from $l p r$ mice exhibited a significant stain reduction compared to the wild type, suggesting the existence of a neuroprotective effect, most probably due to the lack of a functional Fas/CD95 protein (Fig. 1). To clarify whether the identified dying cells were apoptotic, DNA integrity was assessed using TUNEL staining. A decrease in the cell apoptosis in lpr mice treated with KA compared to the wild type (WT) evidenced that the lack of
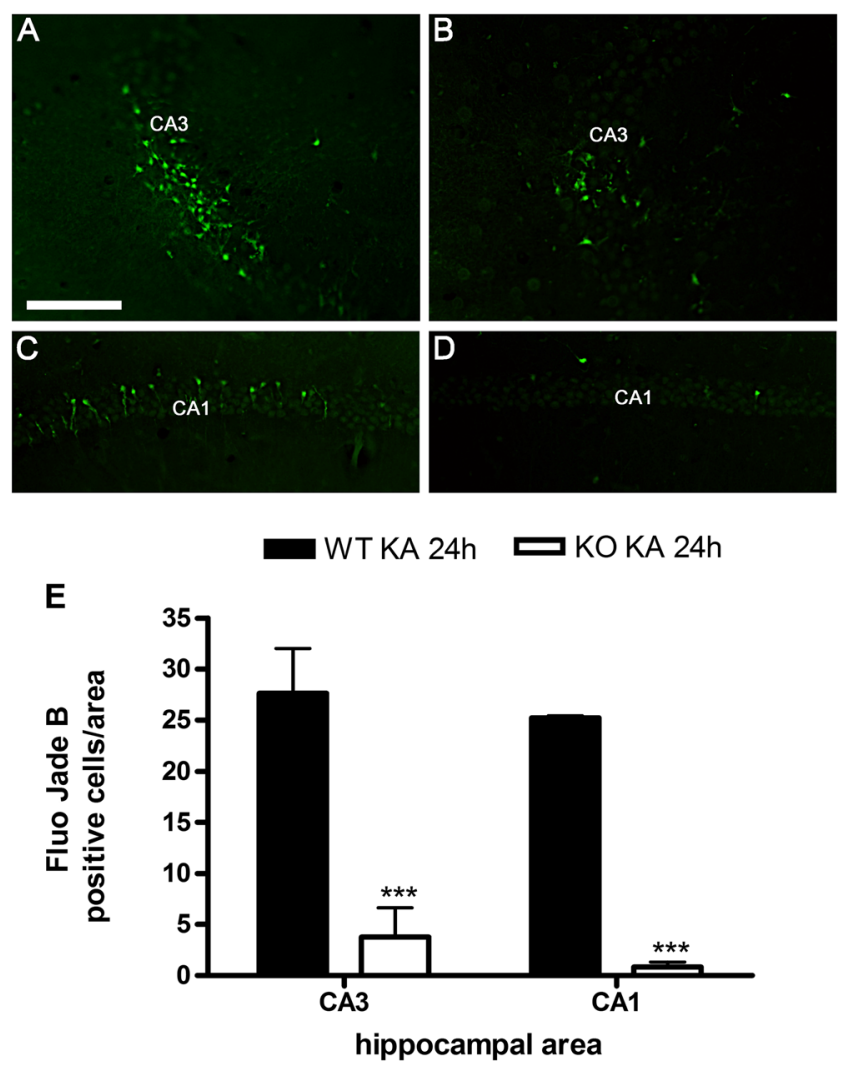

Fig. 1 Neurodegeneration observed by Fluo-Jade B stain in the CA3 and $\mathrm{CA} 1$ regions of the hippocampus after $24 \mathrm{~h}$ of $\mathrm{KA}$ treatment. A decrease of labelled neurons is observed in the CA3 region of kainic acid (KA)treated $l p r$ mice versus wild-type (wt) mice (b vs a). A decrease of labelled neurons is observed in the CA1 region of kainic acid (KA)treated lpr mice versus wild-type (wt) mice (d vs c). Scale bar $100 \mu \mathrm{m}$. (e) A bar graph shows the quantification of positive neurons for Fluoro-Jade $\mathrm{B}$ labelling in the CA3 and CA1 areas of the hippocampus in wild-type and $l p r$ mice. Each point is the mean \pm SEM of three independent experiments, determined in five pictures per experiment $(* * * p<0.001$; with respect to kainic acid-treated wild-type mice)

functionality of the Fas receptor plays an antiapoptotic role (Fig. 2).

\section{Evaluation of Glial Activation in Fas-Deficient $l p r$ Mice Hippocampus After Kainic Acid Treatment}

In the literature, it is widely described that, in addition to neuronal death, the administration of KA produces a glial activation due to excitotoxicity $[4,5]$. This glial reactivity is reflected in a change in the morphology of the astrocytes and microglia. In the case of astrocytes, these acquire a star shape because of the growth of their extensions. In the hippocampal brain areas of the KA-treated wild-type mice, a prominent astrogliosis was observed; thus, we detected an increase in GFAP immunoreactivity as well as shape alterations in astrocytes. The astrocyte activation mediated by KA was not abrogated in lpr mice because the pattern of GFAP immunoreactivity was similar to the wild type (Fig. 3). Microglia are a type 

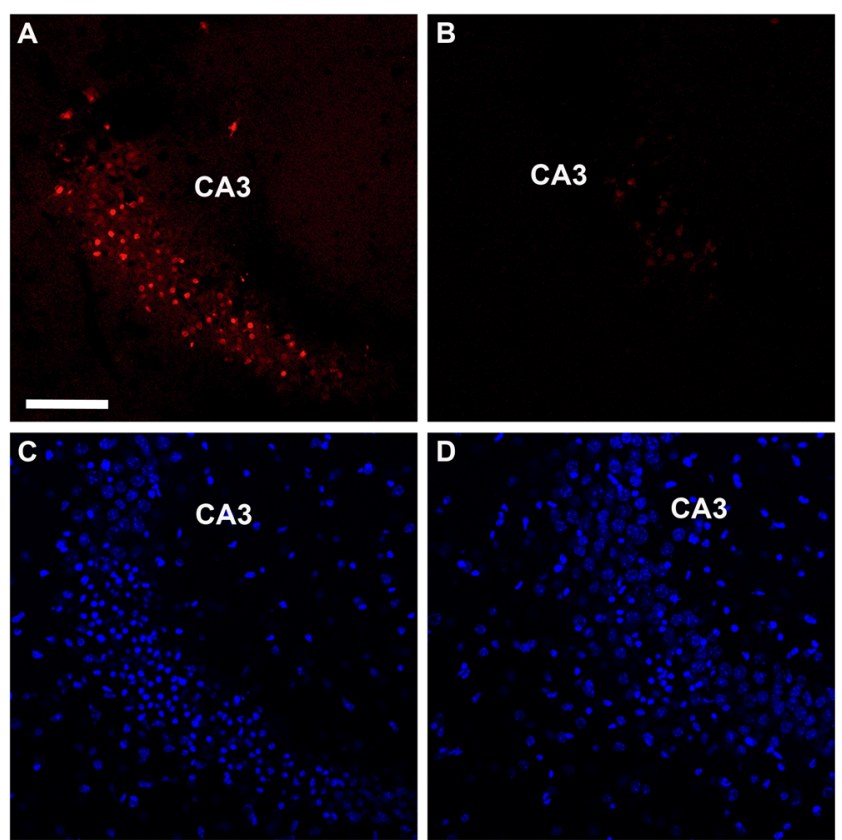

Fig. 2 TUNEL and Hoechst 33342 staining in the CA3 region of the hippocampus from kainic acid-treated wild-type mice (a and $\mathbf{c}$ ) and kainic acid-treated lpr mice (b and d), after $24 \mathrm{~h}$ of treatment. Scale bar $100 \mu \mathrm{m}$

of glial cell acting as the first and main form of active immune defence in the central nervous system, offering an extremely sensitive reaction when neural damage is induced. Thus, microglial activation was analysed in wild-type mice and $l p r$ mice after KA treatment, with an Ibal antibody that allows measurement of the inflammatory response by means of changes in the cell shape of microglial cells, characterised by their amoeboid form. KA treatment evidenced microglial reactivity in the hippocampus, insofar as changes in the morphology of the microglial cells were detected in comparison to saline-treated mice (Fig. 3). Likewise, activated microglial morphology was visualised in lpr mice after KA injection, with the data suggesting that the neuroprotection detected in lpr mice after KA is not mediated by a prevention of glial activation.

Expression of Apoptotic Genes in lpr Mice After Treatment with Kainic Acid

We studied the transcriptional activity of genes involved in the apoptotic response to the exposure to $\mathrm{KA}$ in the lpr mice strain (Table 2). As a first approach, we determined the basal expression of genes in the hippocampus of both $\mathrm{C} 57 / \mathrm{B} 16$ and $l p r$ mice strains (Fig. 4). In fact, results show a clear down-regulation of the fas gene in lpr mice, together with trp53bp2, traf2 and nol3 genes. By contrast, a total of six genes were significantly upregulated: tnf, casp4, casp8, fasl, cflar and cidea. Contrary to what was observed in the C57/B16 wild-type strain, treatment of lpr mice with kainic acid does not produce significant changes in the transcriptional activity of genes related to apoptosis (Table 2). In fact, no significant changes were observed either in the fasl gene or in caspase-4 and caspase- 8 or the executioner caspase-3 genes. In addition to the lack of
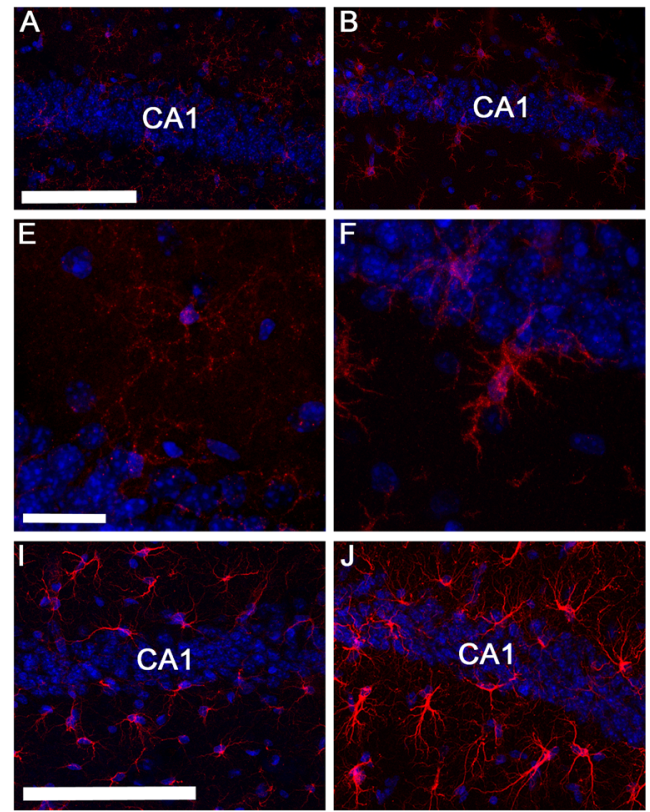

Fig. 3 Microglial and astroglial activation under kainic acid treatment. Iba-1 immunohistochemistry counterstained with Hoechst 33342 in the CA1 region of the hippocampus from saline control wild-type mice (a and $\mathbf{e}$ ), kainic acid-treated wild-type mice (b and $\mathbf{f}$ ), saline control $l p r$ mice (c and $\mathbf{g}$ ) and kainic acid-treated $l p r$ mice (d and h). GFAP
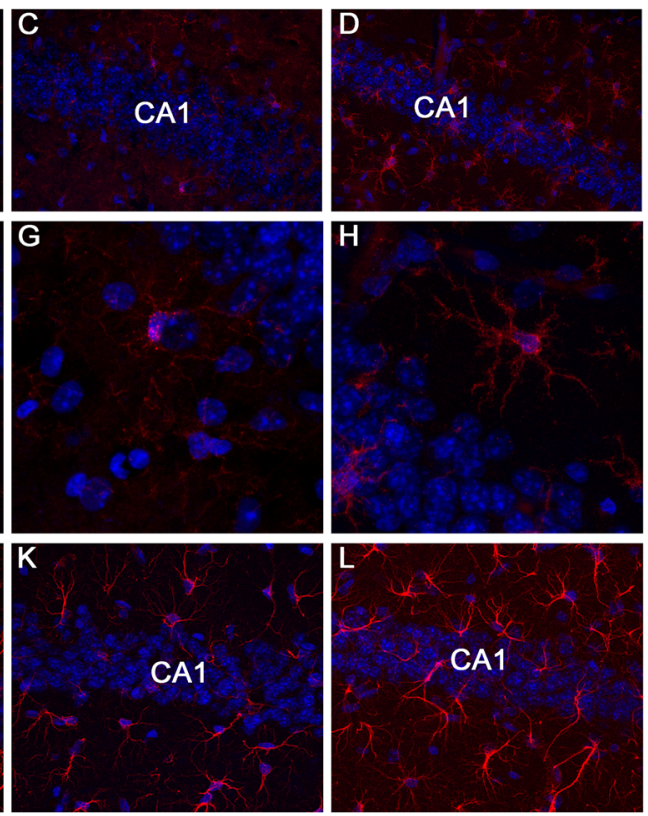

immunohistochemistry counterstained with Hoechst 33342 in the CA1 region of the hippocampus from saline control wild-type mice (i), kainic acid-treated wild-type mice (j), saline control lpr mice (k) and kainic acidtreated $l p r$ mice (I). CA1, CA1 field, hippocampus. Scale bars a-d, $100 \mu \mathrm{m} ; \mathbf{e}-\mathbf{h}, 25 \mu \mathrm{m} ; \mathbf{i}-\mathbf{l}, 100 \mu \mathrm{m}$ 
Table 2 Significant changes in transcriptional activity of genes included in the Mouse Apoptosis RT ${ }^{2}$ Profiler PCR Array, PAMM-012A, from the hippocampus of KA-treated lpr mice, at $12 \mathrm{~h}$. Genes that did not prove to be related, directly or indirectly, to apoptotic processes were excluded from the analysis. By contrast to the observation in the C57/B16 wild-type strain, treatment of $l p r$ mice with KA, at $12 \mathrm{~h}$, did not produce significant changes in transcriptional activity of genes related to apoptosis, neither in fas ligand gene fasl, nor in caspase- 4 and caspase- 8 or the executioner caspase- 3 genes. Any changes were also not observed in atf5

\begin{tabular}{ll}
\hline Gene symbol & $l p r$ KA versus lpr CT \\
\hline tnf & $8.86^{* *}$ \\
bag3 & $5.00^{* * *}$ \\
tnfrsfla & $5.05^{* *}$ \\
fas & $3.14^{* *}$ \\
mcl1 & $2.69^{* *}$ \\
cflar & $1.25^{*}$ \\
casp4 & $0.76^{\mathrm{ns}}$ \\
fasl & $0.95^{\mathrm{ns}}$ \\
ltBr & $1.63^{* *}$ \\
traf2 & $1.47^{* *}$ \\
atf5 & $1.39^{\mathrm{ns}}$ \\
bcl10 & $1.4^{* *}$ \\
casp3 & $1.11^{\mathrm{ns}}$ \\
casp8 & $1.09^{\mathrm{ns}}$ \\
nol3 & $0.48^{* *}$ \\
cidea & $0.85^{\mathrm{ns}}$ \\
cradd & $0.43^{*}$ \\
trp53bp2 & $0.56^{* * *}$ \\
\hline
\end{tabular}

Asterisks different levels of significance

$n s$ non significant (non statistical differences)

activity in cytoplasmic caspase genes, the cell deathinducing DNA fragmentation factor gene, cidea, showed no significant change in transcriptional activity. Nor were any changes observed in the atf5 gene. Therefore, it can be concluded that Fas signalling is involved in kainic acid-induced apoptosis in hippocampal cells (Fig. 5).

\section{Ipr CT vs WT CT}

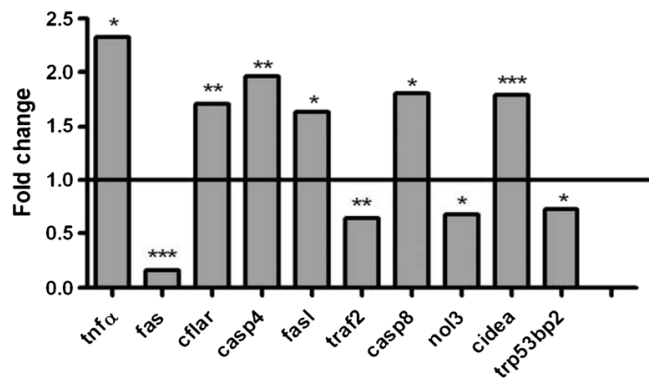

Fig. 4 Significant changes in the transcriptional activity of genes included in the Mouse Apoptosis RT ${ }^{2}$ Profiler PCR Array, PAMM-012A, from the hippocampus of both C57/B16 wild-type and lpr mice. In lpr mice, a clear down-regulation of the fas, $\operatorname{trp} 53 \mathrm{bp} 2, \operatorname{traf} 2$ and nol3 genes is observed when compared with C57/B16. By contrast, a total of six genes were significantly up-regulated: tnf, casp4, casp8, fasl, cflar and cidea

\section{Discussion}

In the present paper, we report evidence of a novel role for Fas in the apoptosis of hippocampal neurons after treatment with the neurotoxin KA. To provide a mechanistic basis for these findings, we demonstrated that $l p r$ mice treated with this neurotoxin exhibit a significant reduction in neuronal cell death and apoptosis compared with wild-type mice.

Real-time PCR array analysis showed that KA significantly regulated 23 apoptosis-related genes (fold change $\geq \pm 1.5$ and $P$ value 0.05 ). It is well established that the extrinsic signalling pathway that initiates apoptosis involves death receptor members of the Tnf receptor gene superfamily [21, 23, 26, 42, 46, 47]. Our study demonstrated that Fas, Tnfrl, Fasl and Tnf genes were highly expressed in the hippocampus following KA treatment in wild-type animals. In addition, our findings also prove an overexpression of the Casp 8 gene that encodes an initiator caspase and the Casp3 gene that encodes an effector caspase. However, it is noteworthy that KA treatment at $12 \mathrm{~h}$ does not promote significant changes in the expression of key genes involved in the intrinsic apoptotic pathway such as caspase-9 and Apaf1, among others. These data suggest that the cell death observed at $24 \mathrm{~h}$ of KA treatment may be due mainly to the activation of genes involved in the extrinsic pathway of apoptosis.

Several reports have demonstrated that the expression of the Fas receptor gene is induced after neuropathological lesions including those produced by KA [23-25, 38, 48]. With the aim of reporting more data in the involvement of the Fas signalling pathway in the mechanisms of neuronal death induced by excitotoxicity, we performed a treatment with KA in $l p r$ mice. Previous studies have described that these mice exhibit neuroprotection against traumatic brain injury (TBI), in preclinical models of Parkinson's disease (MPTP) and amyotrophic lateral sclerosis (ALS), and also models of ischemia, demonstrating the involvement of the Fas pathway in neuronal death [24, 25, 29, 32, 41, 47, 49]. We observed a significant decrease in neurodegeneration in the hippocampus of the KA-treated lpr mice compared to the wild type, so the results strongly suggest that the lack of Fas receptor functionality plays a neuroprotective role following KA administration.

In the model of KA-induced excitotoxicity, it is widely demonstrated that there is clear astroglial and microglial activation [4, 5, 50]. Here, in the present study, we have also observed glial reactivity after KA treatment in the hippocampus of wild-type mice. In the case of the lpr mice, although there was a significant reduction in neuronal death, the glial reactivity induction was similar to that of wild-type mice, indicating that while the Fas pathway prevents neuronal death, it is not involved in the regulation of neuroinflammatory processes. Likewise, KA-induced glial activation may occur through mechanisms independent of Fas [27]. 
Fig. 5 Drawing of the 23 genes identified by the Mouse Apoptosis RT ${ }^{2}$ Profiler PCR Array, PAMM-012A, with a significative differential expression (fold change $\geq \pm 1.5$ and $P$ value 0.05 ) between KA wild-type treated and untreated mice, at $12 \mathrm{~h}$. Fas/FasL interaction induces caspase- 8 and caspase- 3 activity. Caspase- 3 is a key mediator of apoptosis in neuronal cells. This pathway is blocked in the lpr mice fold change $\geq \pm 1.5$

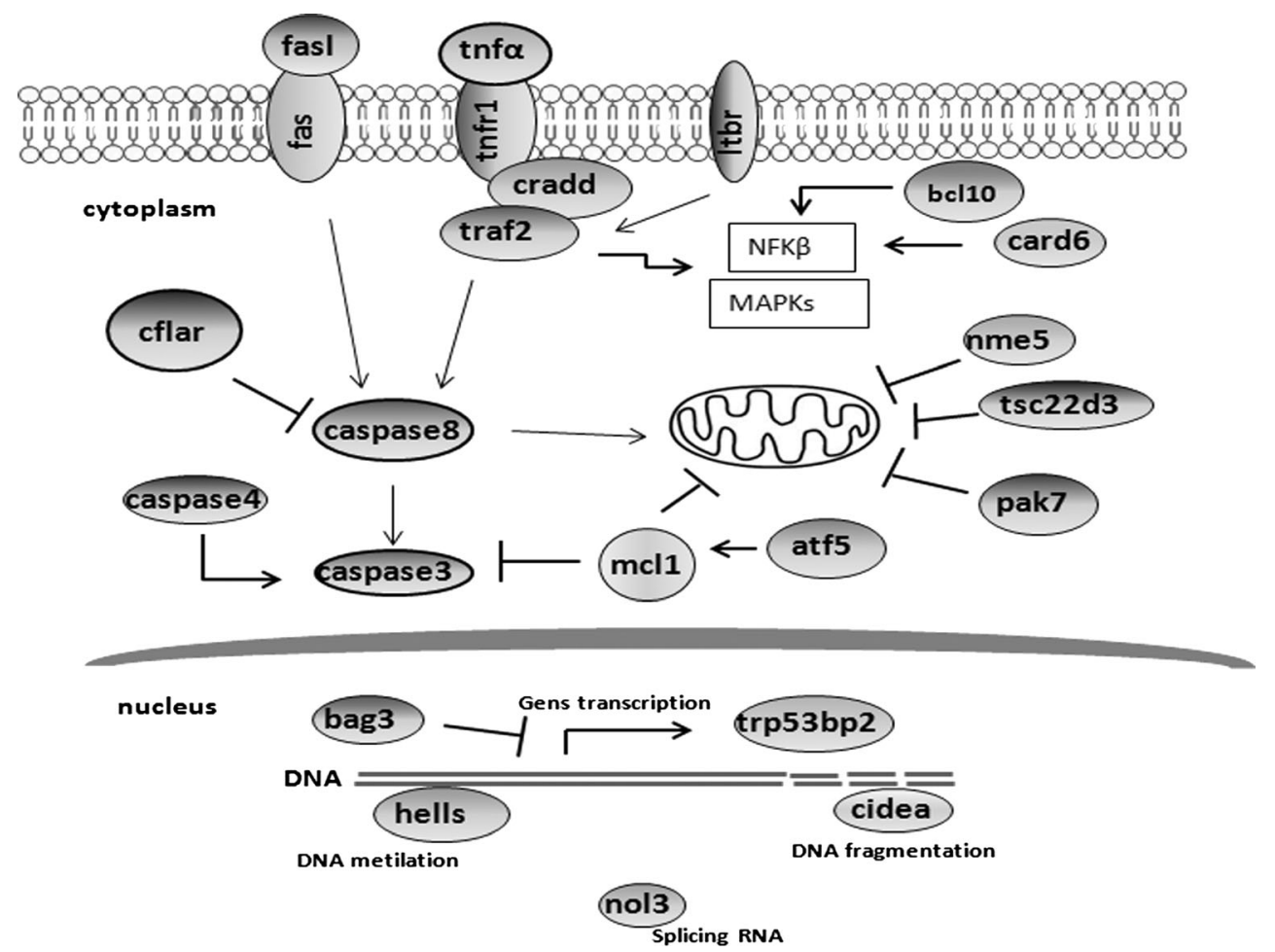

Because reduced neuronal death was observed in $l p r$ mice following KA treatment, we analysed the differentially expressed apoptosis-related genes detected, comparing wildtype KA-treated mice to untreated mice. For this, first of all, we compared the gene expression levels between the two genotypes (wild type and $l p r$ ). The $l p r$ mice showed higher expression levels of genes encoding the death receptor ligands (FasL and TNF): caspase- 8 that codifies an essential protease for the extrinsic apoptotic pathway, caspase-4 encoding a caspase that plays a role in inflammation, cflar that codifies an apoptotic regulator protein that lacks caspase activity and appears to be itself cleaved into two peptides by Caspase- 8 and the pro-apoptotic cidea gene $[42,51]$. Furthermore, a down-regulation of the nol3 (nucleolar protein 3) gene was detected, which encodes the antiapoptotic protein ARC (apoptosis repressor with a caspase recruitment domain) that interacts with Caspase- 2 and Caspase- 8 and inhibits receptormediated apoptosis. In addition, nol3 does not play a key role in $l p r$ neuroprotection, because we did not find an increase in gene expression after $l p r$ mice KA treatment.

A down-regulation was also detected in traf 2 encoding TRAF2 protein required for TNF-alpha-mediated activation of MAPK $8 / \mathrm{JNK}$ and NF- $\mathrm{kB}$ and in trp53bp 2 that codifies a pro-apoptotic member of a family of $\mathrm{p} 53$ binding proteins [52]. All these data indicate that the $l p r$ mice genotype does not respond to a neuroprotective profile.

However, after KA administration, we found that some genes implicated in the death pathway did not show significant changes in expression in $l p r$ mice compared to WT, such as fasl, decreasing the FasR/FasL interaction: caspase-3 and caspase-8, the main caspases involved in the extrinsic apoptotic pathway; caspase-4 involved in inflammatory processes; cidea that codifies a apoptotic protein activator and pim 2 that codifies an enzyme serine/threonine kinase that has roles in apoptosis and regulation of signal transduction cascades $[18,25,52-58]$. This fact suggests that the expression of these genes could be regulated by the expression of $f a s$ in the model of KA-induced neurotoxicity, indicating that they play an important role in the apoptotic cascade in the excitotoxic process.

In agreement with Torres-Peraza et al. [59], we also observed an increase of atf5 gene expression mediated by KAinduced seizures; interestingly, a neuronal increase in atf5 expression has a neuroprotective role [59]. It has been proposed that the neuroprotective effects of ATF5 are mediated by the transcriptional induction of some proteins with a neuroprotective effect, such as Hsp27, Bcl2 and Mcl1 [58-61]. Likewise, in our study, we found a significant up-regulation in $m c l l$, which was also up-regulated in other models of status epilepticus, such as pilocarpine administration in rodents [62].

Furthermore, in our study, after KA treatment in $l p r$ mice compared to WT, we observed a non-significant increase in atf5, possibly resulting from the decrease in apoptotic neuronal death that occurs in $l p r$ KA-treated mice. At the same time, other genes showed similar changes in gene expression profiles between the two genotypes, including bag3, mcll, traf 2 and bcl10. Interestingly, these genes encode proteins that confer antiapoptotic neuroprotection, which could account 
for the increase in expression in both WT and lpr mice after KA treatment [62]. Therefore, our results suggest neuroprotective effects in $l p r$ mice after KA administration based on genes that are not activated in this model. Furthermore, these genes could be considered as potential therapeutic drug targets in neurodegenerative diseases. In this line, recent data have demonstrated that a mouse with neuronal caspase- 8 deficiency was protected against neuronal injury caused by KAinduced excitotoxicity [63]. The apoptotic prevention observed in $l p r$ mice after KA administration may be at least partly attributable to a decrease in caspase- 8 , which is also involved in caspase- 3 activation. At the same time, the differential gene expression observed in some apoptosis-related genes between untreated genotypes should be intensively studied to determine their functional significance in the process of neuroprotection observed in mice deficient in the Fas receptor.

In conclusion, in the present work, we report evidence of the key role played by the Fas/FasL-mediating apoptosis of neurons in contributing to the process of KAinduced neurodegeneration and affecting neuronal function and survival (Fig. 5). Interestingly, FasR-deficient mice exhibit significantly reduced cell death, although inflammatory cells (microglia/macrophages) and the level of GFAP when compared with the wild type were unchanged. Thus, we propose that targeting the Fas receptor could provide a compelling rationale for therapeutic strategies against neurodegeneration but not against neuroinflammation.

Acknowledgments This work was supported by Centro de Investigación Biomédica en Red de Enfermedades Neurodegenerativas (CiberNed-Instituto de Salud Carlos III) and by grants from Ministerio de Ciencia (MICINN, MINECO) SAF2009-08233 and SAF2012-34177 and Fundación Ramón Areces to JJL. Grant 2009/SGR00853 from the Generalitat de Catalunya (Autonomous Government of Catalonia) and grants BFU2010-19119/BFI to CA, SAF2011-23631 to AC, and SAF2012-39852-C02-01 to MP from the Spanish Ministerio de Ciencia (MICINN, MINECO) also supported the study. Grant 0177594 from CONACYT (Mexico) was awarded to CBZ.

Conflict of Interest The authors declare no conflict of interest.

\section{References}

1. Beal MF (1998) Excitotoxicity and nitric oxide in Parkinson's disease pathogenesis. Ann Neurol 44(Suppl 1):S110-S114

2. Boll MC, Alcaraz-Zubeldia M, Rios C (2011) Medical management of Parkinson's disease: focus on neuroprotection. Curr Neuropharmacol 9:350-359

3. Mehta A, Prabhakar M, Kumar P, Deshmukh R, Sharma PL (2013) Excitotoxicity: bridge to various triggers in neurodegenerative disorders. Eur J Pharmacol 698:6-18

4. Ben-Ari Y, Cossart R (2000) Kainate, a double agent that generates seizures: two decades of progress. Trends Neurosci 23:580-587
5. Benkovic SA, O'Callaghan JP, Miller DB (2004) Sensitive indicators of injury reveal hippocampal damage in $\mathrm{C} 57 \mathrm{BL} / 6 \mathrm{~J}$ mice treated with kainic acid in the absence of tonic-clonic seizures. Brain Res 1024: 59-76

6. de Lemos L, Junyent F, Verdaguer E, Folch J, Romero R, Pallàs M, Ferrer I, Auladell C, Camins A (2010) Differences in activation of ERK1/2 and p38 kinase in Jnk3 null mice following KA treatment. J Neurochem 114:1315-1322

7. Fisahn A (2005) Kainate receptors and rhythmic activity in neuronal networks: hippocampal gamma oscillations as a tool. J Physiol 562: $65-72$

8. Tripathi PP, Santorufo G, Brilli E, Borrelli E, Bozzi Y (2011) Kainic acid-induced seizures activate GSK-3 $\beta$ in the hippocampus of D2R $-/-$ mice. Neuroreport 23:846-850

9. Wang Q, Yu S, Simonyi A, Sun GY, Sun AY (2005) Kainic acidmediated excitotoxicity as a model for neurodegeneration. Mol Neurobiol 31:3-16

10. Engel T, Hatazaki S, Tanaka K, Prehn JH, Henshall DC (2010) Deletion of Puma protects hippocampal neurons in a model of severe status epilepticus. Neuroscience 168:443-450

11. Engel T, Murphy BM, Hatazaki S, Jimenez-Mateos EM, Concannon CG, Woods I et al (2010) Reduced hippocampal damage and epileptic seizures after status epilepticus in mice lacking proapoptotic Puma. FASEB J 24:853-861

12. Crespo-Biel N, Canudas AM, Camins A, Pallàs M (2007) Kainate induces AKT, ERK and cdk5/GSK3beta pathway deregulation, phosphorylates tau protein in mouse hippocampus. Neurochem Int 50: 435-442

13. Grabenstatter HL, Del Angel YC, Carlsen J, Wempe MF, White AM et al (2014) The effect of STAT3 inhibition on status epilepticus and subsequent spontaneous seizures in the pilocarpine model of acquired epilepsy. Neurobiol Dis 62:73-85

14. Koeller HB, Ross ME, Glickstein SB (2008) Cyclin D1 in excitatory neurons of the adult brain enhances kainate-induced neurotoxicity. Neurobiol Dis 31:230-241

15. Murphy BM, Engel T, Paucard A, Hatazaki S, Mouri G, Tanaka K et al (2010) Contrasting patterns of Bim induction and neuroprotection in Bim-deficient mice between hippocampus and neocortex after status epilepticus. Cell Death Differ 17:459-468

16. Verdaguer E, Jiménez A, Canudas AM, Jordà EG, Sureda FX et al (2004) Inhibition of cell cycle pathway by flavopiridol promotes survival of cerebellar granule cells after an excitotoxic treatment. J Pharmacol Exp Ther 308:609-616

17. Algeciras-Schimnich A, Barnhart BC, Peter ME (2002) Apoptosisindependent functions of killer caspases. Curr Opin Cell Biol 14: 721-726

18. Galluzzi L, Blomgren K, Kroemer G (2009) Mitochondrial membrane permeabilization in neuronal injury. Nat Rev Neurosci 10:481494

19. Kumar S (2007) Caspase function in programmed cell death. Cell Death Differ 14:32-43

20. Le-Niculescu H, Bonfoco E, Kasuya Y, Claret FX, Green DR, Karin M (1999) Withdrawal of survival factors results in activation of the JNK pathway in neuronal cells leading to Fas ligand induction and cell death. Mol Cell Biol 19:751-763

21. Henshall DC, Bonislawski DP, Skradski SL, Lan JQ, Meller R, Simon RP (2001) Cleavage of bid may amplify caspase-8-induced neuronal death following focally evoked limbic seizures. Neurobiol Dis 8:568-580

22. Henshall DC, Araki T, Schindler CK, Shinoda S, Lan JQ, Simon RP (2003) Expression of death-associated protein kinase and recruitment to the tumor necrosis factor signaling pathway following brief seizures. J Neurochem 86:1260-1270

23. Tan Z, Levid J, Schreiber SS (2001) Increased expression of Fas (CD95/APO-1) in adult rat brain after kainate-induced seizures. Neuroreport 12:1979-1982 
24. Dzietko M, Boos V, Sifringer M, Polley O, Gerstner B, Genz K et al (2008) A critical role for Fas/CD-95 dependent signaling pathways in the pathogenesis of hyperoxia-induced brain injury. Ann Neurol 64:664-673

25. Ethell DW, Buhler LA (2003) Fas ligand-mediated apoptosis in degenerative disorders of the brain. J Clin Immunol 23:363-370

26. Felderhoff-Mueser U, Buhrer C, Groneck P, Obladen M, Bartmann P, Heep A (2003) Soluble Fas (CD95/Apo-1), soluble Fas ligand, and activated caspase 3 in the cerebrospinal fluid of infants with posthemorrhagic and nonhemorrhagic hydrocephalus. Pediatr Res 54:659-664

27. Grosjean MB, Lenzlinger PM, Stahel PF, Yatsiv I, Shohami E, Trentz $O$ et al (2007) Immunohistochemical characterization of Fas (CD95) and Fas Ligand (FasL/CD95L) expression in the injured brain: relationship with neuronal cell death and inflammatory mediators. Histol Histopathol 22:235-250

28. Jia J, Guan D, Zhu W, Alkayed NJ, Wang MM, Hua Z, Xu Y (2009) Estrogen inhibits Fas-mediated apoptosis in experimental stroke. Exp Neurol 215:48-52

29. Martin-Villalba A, Herr I, Jeremias I, Hahne M, Brandt R, Vogel J et al (1999) CD95 ligand (Fas-L/APO-1L) and tumor necrosis factorrelated apoptosis-inducing ligand mediate ischemia-induced apoptosis in neurons. J Neurosci 19:3809-3817

30. Chu JL, Drappa J, Parnassa A, Elkon KB (1993) The defect in Fas mRNA expression in MRL/lpr mice is associated with insertion of the retrotransposon, ETn. J Exp Med 178:723-730

31. Graham EM, Sheldon RA, Flock DL, Ferriero DM, Martin LJ et al (2004) Neonatal mice lacking functional Fas death receptors are resistant to hypoxic-ischemic brain injury. Neurobiol Dis 17:89-98

32. Niu FN, Zhang X, Hu XM, Chen J, Chang LL, Li JW et al (2012) Targeted mutation of Fas ligand gene attenuates brain inflammation in experimental stroke. Brain Behav Immun 26:61-71

33. Safa AR (2012) c-FLIP, a master anti-apoptotic regulator. Exp Oncol 34:176-184

34. Hayley S, Crocker SJ, Smith PD, Shree T, Jackson-Lewis V, Przedborski S, Mount M et al (2004) Regulation of dopaminergic loss by Fas in a 1-methyl-4-phenyl-1,2,3,6-tetrahydropyridine model of Parkinson's disease. J Neurosci 24:2045-2053

35. Landau AM, Luk KC, Jones ML, Siegrist-Johnstone R, Young YK, Kouassi E et al (2005) Defective Fas expression exacerbates neurotoxicity in a model of Parkinson's disease. J Exp Med 202:575-581

36. Mogi M, Harada M, Kondo T, Mizuno Y, Narabayashi H, Riederer P, Nagatsu T (1996) The soluble form of Fas molecule is elevated in parkinsonian brain tissues. Neurosci Lett 220:195-198

37. Bi FF, Xiao B, Hu YQ, Tian FF, Wu ZG, Ding L, Zhou XF (2008) Expression and localization of Fas-associated proteins following focal cerebral ischemia in rats. Brain Res 1191:30-38

38. Ethell DW, Kinloch R, Green DR (2002) Metalloproteinase shedding of Fas ligand regulates beta-amyloid neurotoxicity. Curr Biol 12: $1595-1600$

39. Millet P, Lages CS, Haik S, Nowak E, Allemand I, Granotier C, Boussin FD (2005) Amyloid-beta peptide triggers Fas-independent apoptosis and differentiation of neural progenitor cells. Neurobiol Dis 19:57-65

40. Morishima Y, Gotoh Y, Zieg J, Barrett T, Takano H et al (2001) Betaamyloid induces neuronal apoptosis via a mechanism that involves the c-Jun N-terminal kinase pathway and the induction of Fas ligand. J Neurosci 21:7551-7560

41. Shah RS, Lee HG, Xiongwei Z, Perry G, Smith MA, Castellani RJ (2008) Current approaches in the treatment of Alzheimer's disease. Biomed Pharmacother 62:199-207

42. Puri V, Ranjit S, Konda S, Nicoloro SM, Straubhaar J, Chawla A et al (2008) Cidea is associated with lipid droplets and insulin sensitivity in humans. Proc Natl Acad Sci U S A 105:7833-7838

43. Watanabe-Fukunaga R, Brannan CI, Itoh N, Yonehara S, Copeland NG et al (1992) The cDNA structure, expression, and chromosomal assignment of the mouse Fas antigen. J Immunol 148:1274-1279
44. Watanabe-Fukunaga R, Brannan CI, Copeland NG, Jenkins NA, Nagata S (1992) Lymphoproliferation disorder in mice explained by defects in Fas antigen that mediates apoptosis. Nature 356:314-317

45. Leite JP, Garcia-Cairasco N, Cavalheiro EA (2002) New insights from the use of pilocarpine and kainate models. Epilepsy Res 50: 93-103

46. Hartmann A, Mouatt-Prigent A, Faucheux BA, Agid Y, Hirsch EC (2002) FADD: a link between TNF family receptors and caspases in Parkinson's disease. Neurology 58:308-310

47. Su JH, Anderson AJ, Cribbs DH, Tu C, Tong L et al (2003) Fas and Fas ligand are associated with neuritic degeneration in the $\mathrm{AD}$ brain and participate in beta-amyloid-induced neuronal death. Neurobiol Dis $12: 182-193$

48. Ferrer I, Puig B, Krupinsk J, Carmona M, Blanco R (2001) Fas and Fas ligand expression in Alzheimer's disease. Acta Neuropathol 102: $121-131$

49. Williams CD, McGill MR, Farhood A, Jaeschke H (2013) Fas receptor-deficient lpr mice are protected against acetaminophen hepatotoxicity due to higher glutathione synthesis and enhanced detoxification of oxidant stress. Food Chem Toxicol 58:228-235

50. Puig B, Ferrer I (2002) Caspase-3-associated apoptotic cell death in excitotoxic necrosis of the entorhinal cortex following intraperitoneal injection of kainic acid in the rat. Neurosci Lett 321:182-186

51. Nohara N, Koseki T, Chen S, Wu X, Nunez G (1998) CIDE, a novel family of cell death activators with homology to the $45 \mathrm{kDa}$ subunit of the DNA fragmentation factor. EMBO J 17:2526-2533

52. Kampa KM, Bonin M, Lopez CD (2009) New insights into the expanding complexity of the tumor suppressor ASPP2. Cell Cycle 8:2871-2876

53. Alvarado Y (2012) The PIM kinases in hematological cancers. Expert Rev Hematol 5:81-96

54. Benkovic SA, O'Callaghan JP, Miller DB (2006) Regional neuropathology following kainic acid intoxication in adult and aged C57BL/ 6J mice. Brain Res 1070:215-231

55. Chuang YC, Lin JW, Chen SD, Lin TK, Liou CW, Lu CH, Chang WN (2009) Preservation of mitochondrial integrity and energy metabolism during experimental status epilepticus leads to neuronal apoptotic cell death in the hippocampus of the rat. Seizure 18:420-428

56. Diwakarla S, Nagley P, Hughes ML, Chen B, Beart PM (2009) Differential insult-dependent recruitment of the intrinsic mitochondrial pathway during neuronal programmed cell death. Cell Mol Life Sci 66:156-172

57. Engel T, Henshall DC (2009) Apoptosis, Bcl-2 family proteins and caspases: the $\mathrm{ABCs}$ of seizure-damage and epileptogenesis? Int $\mathrm{J}$ Physiol Pathophysiol Pharmacol 1:97-115

58. Wei XW, Yan H, Xu B, Wu YP, Li C, Zhang GY (2012) Neuroprotection of co-activation of GABA receptors by preventing caspase-3 denitrosylation in KA-induced seizures. Brain Res Bull 88:617-623

59. Torres-Peraza JF, Engel T, Martín-Ibáñez R, Sanz-Rodríguez A, Fernández-Fernández MR et al (2013) Protective neuronal induction of ATF5 in endoplasmic reticulum stress induced by status epilepticus. Brain 136:1161-1176

60. Wang H, Lin G, Zhang Z (2007) ATF5 promotes cell survival through transcriptional activation of $\mathrm{Hsp} 27$ in $\mathrm{H} 9 \mathrm{c} 2$ cells. Cell Biol Int 31:1309-1315

61. Dluzen D, Li G, Tacelosky D, Moreau M, Liu DX (2011) BCL-2 is a downstream target of ATF5 that mediates the prosurvival function of ATF5 in a cell type-dependent manner. J Biol Chem 286:7705-7713

62. Mori M, Burgess DL, Gefrides LA, Foreman PJ, Opferman JT, Korsmeyer SJ et al (2004) Expression of apoptosis inhibitor protein Mcl1 linked to neuroprotection in CNS neurons. Cell Death Differ 11:1223-1233

63. Krajewska M, You Z, Rong J, Kress C, Huang X et al (2011) Neuronal deletion of caspase 8 protects against brain injury in mouse models of controlled cortical impact and kainic acid-induced excitotoxicity. PLoS ONE 6:e24341 